\title{
Modification algorithm of Cubic B-spline curve interpolation
}

\author{
Wan-Jun Zhang ${ }^{1,2,3, a}$, Shan-Ping Gao ${ }^{1, b}$ Su-Jia Zhang ${ }^{1, c}$ \&Feng Zhang ${ }^{2, d}$ \\ 1 Quanzhou Institute of Information Engineering, Guangzhou 510000, China \\ 2 School of Mechanical Engineering, Xian Jiao tong University, Xi'an 7410049, China
}

3 Lanzhou Industry and Equipment Co. ,Ltd. , Lanzhou 730050, China

\begin{abstract}
Based on cubic B-Spline curve mathematical properties, theoretical analysis the cubic B-Spline curve recursive formula of Taylor development of first-order, derivation of two order in the interpolation cycle under the condition of certain interpolation increment only and interpolation speed, change the interpolation increments can be amended cubic times B-Spline curves purpose. So this paper presents such a modified interpolation algorithm, which is combined with specific examples of matlab7.0 verifies that the algorithm is correct; it is consistent with a cubic B-Spline curve interpolation requirements.
\end{abstract}

Keywords:Cubic B-Spline curve; CNC system; Interpolation algorithm .

\section{Introduction}

In modern NC system, Non-Uniform Rational B-spline (NURBUS ) has been wider and wide used in surface and curve, also NURBS is a popular free curve and surface expression, but the generation principle and expression is relatively complex, and cubic B-Spline curve because can meet the processing problems of general needs and the computation is simple [1], the cubic B-spline curve is widely used in engineering method for curve construction and engineering and technical personnel to solve the problems associated with the preferred. At present, more and more researchers have researched interpolation algorithms for parametric B-spline curves. Shpitalni et al [3-5] research the curves of parametric algorithms on the basis of Taylor's first-order or second-order Taylor's expansion. Literature [6-8] give some NURBS interpolation algorithms, which not only makes NC programming complicated, but also cannot meet the high-speed and high-accuracy NC machine tool require-ments of CNC systems.

On the basis of the research above, in this paper, an modification algorithm of Cubic B-spline curve interpolation was introduced. first-order approximation of Taylor's expansion and tow-order approximation of Taylor's expansion Can be simplified into first-order approximation of Taylor's expansion. So, The interpolation of Cubic B-spline curve only interpolation speed, change the interpolation increments can be amended cubic times B-Spline curves purpose. By using matlab7.0 , the simulation results show that the proposed Cubic B-spline curve interpolator algorithm is correct ,also meet the NC machine tool require-ments of NC systems.

\section{Cubic B-Spline curve mathematical definition}

In this note, B-Spline curve is used to represent a parametric adaptive of curve, and it is introduced first. Supposed $p(u)$ can be represented B-Spline curve. While B-Spline curve [9] are parametrically mathematical definition by the following Eq.(1).

$$
p(u)=\sum_{i=0}^{n} \omega_{i} d_{i, k}(u) / \sum_{i=0}^{n} \omega_{i} N_{i, k}(u)=\sum_{i=0}^{n} p_{i} N_{i, k}(u)
$$

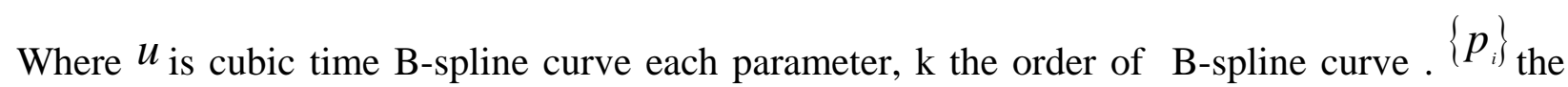
control points and $\left\{\omega_{i}\right\}$ the element of the weight vector were formed a group of weighted control 
points $p_{i}^{\omega}=\left(x_{i} \omega_{i}, y_{i} \omega_{i}, Z_{i} \omega_{i}\right)$, when $\omega_{i}^{=1}$, B-spline curve is hyper plane projection corresponding to the coordinates of the point.We can get $p_{i}^{\omega}=\left(x_{i}, y_{i}, z_{i}\right) . N_{i, k}(u)$ is the blending function .

$$
N_{i, k}(u)=\left\{\begin{array}{l}
N_{i, 0}=\left\{\begin{array}{c}
1, u_{i} \leq u \leq u_{i+1} \\
0, \text { otherwise }
\end{array}\right. \\
N_{i, k}=\frac{u-u_{i}}{u_{i+k}-u_{i}} N_{i, k-1}(u) \\
\quad+\frac{u i+k+1-u}{u_{i+k+1}-u_{i+1}} N_{i+1, k-1}(u) \\
\text { define: } \frac{0}{0}=0
\end{array}\right.
$$

\section{Cubic B-Spline curve modification algorithm}

By using the second-order Taylor series of ${ }^{u(t)}$ at $t=t_{i}$,the next parameter position $u_{i}$ can be determined as

$$
u_{i+1}=u_{i}+\left.\frac{d u}{d t}\right|_{t=t_{i}} T+\left.\frac{1}{2} \frac{d^{2} u}{d t^{2}}\right|_{t=t_{i}} T^{2}+\text { H.O.T. }
$$

Where $T$ is the sampling period and H.O.T. represents the high order truncation error.

The feed speed $V\left(\boldsymbol{u}_{i}\right)$ along B-Spline curve is defined by

$$
V\left(\boldsymbol{u}_{1}\right)=\left\|\frac{d p(u)}{d t}\right\|=\left\|\frac{d p(u)}{d u}\right\| \frac{d u}{d t}
$$

By substituting $\frac{d u}{d t}$ into Eq.(5)

$$
\frac{d u}{d t}=\frac{V(t)}{\left\|\frac{d p(u)}{d t}\right\|}
$$

Also, the first-order interpolation equation for B-Spline curve is obtained by substituting Eq.(3)into Eq.(6) , The first-order approximation of Taylor's expansion for calculating $\boldsymbol{u}_{i+1}$ is as follows Eq.(6) can be represented as follows

$$
u_{i+1}=u_{i}+\frac{V\left(u_{i}\right) \cdot T}{\left|\frac{d p(u)}{d t}\right|} u_{u=u_{i}}=\frac{V\left(u_{i}\right) \cdot T}{\sqrt{{x_{i}^{\prime}}^{2}+y^{\prime^{2}}+{z^{\prime}}_{i}^{2}}}
$$

Where $T$ is the interval (sec), $\left(\boldsymbol{x}_{i}, y_{i}, z_{i}\right)$ is interpolation coordinates.

The second-order approximation of Taylor's expansion is

$$
u_{i+1}=u_{i}+\frac{V\left(u_{i}\right) \cdot T}{\left|\frac{d p(u)}{d t}\right| u=u_{i}}-\frac{V^{2} \cdot T^{2}\left(\frac{d u}{d t} \cdot \frac{d^{2} p(u)}{d t^{2}}\right) \mid u=u_{i}}{2 \cdot\left|\frac{d p(u)}{d t}\right| u=u_{i}}
$$


So, The second derivative $\mathrm{u}$ with $\mathrm{t}$ is obtained as $\frac{\mathrm{d}^{2} u}{\mathrm{~d} t^{2}} \mid t=t$

$\left.\frac{\mathrm{d}^{2} u}{\mathrm{~d} t^{2}}\right|_{t=t_{i}}=-\frac{v^{2}}{\left\|\frac{\mathrm{d} p(u)}{\mathrm{d} t}\right\|^{4} u=u_{i}}\left(\frac{\mathrm{d} p(u)}{\mathrm{d} u}\left|u=u_{i} \cdot \frac{\mathrm{d} p^{2}(u)}{\mathrm{d} u^{2}}\right| u=u_{i}\right)$

Set B curve is expressed as $p(u)$, the curve $u=u_{i}$ parameters for b curvature

$$
b=\left(\frac{\mathrm{d} p(u)}{\mathrm{d} u}\left|u=u_{i} \cdot \frac{\mathrm{d} p^{2}(u)}{\mathrm{d} u^{2}}\right| u=u_{i}\right) \frac{1}{\left\|\frac{\mathrm{d} p(u)}{\mathrm{d} t}\right\|^{2} u=u_{i}}
$$

Also , the curvature radius $R_{i}$

$$
\begin{aligned}
& \begin{array}{c}
R_{i}=\frac{1}{b}=\frac{1}{\left(\frac{\mathrm{d} p(u)}{\mathrm{d} u}\left|u=u_{i} \cdot \frac{\mathrm{d} p^{2}(u)}{\mathrm{d} u^{2}}\right| u=u_{i}\right) \frac{1}{\left\|\frac{\mathrm{d} p(u)}{\mathrm{d} t}\right\|^{2} u=u_{i}}}=\frac{\left\|\frac{\mathrm{d} p(u)}{\mathrm{d} t}\right\|^{2} u=u_{i}}{\left(\frac{\mathrm{d} p(u)}{\mathrm{d} u}\left|u_{i}=u_{i} \cdot \frac{\mathrm{d} p^{2}(u)}{\mathrm{d} u^{2}}\right| u\right.} \\
\text { When } R_{i} \rightarrow \infty, b \rightarrow 0, \\
\text { Where } \\
\frac{\mathrm{d}^{2} u}{\mathrm{~d} t^{2} \mid}{ }_{t=t_{i}}=0
\end{array} \\
& u_{i+1}=u_{i}+\frac{V\left(u_{i}\right) \cdot T}{\left|\frac{d p(u)}{d t}\right|} u_{u=u_{i}}=\frac{V\left(u_{i}\right) \cdot T}{\sqrt{{x^{\prime}}_{i}{ }^{2}+y^{\prime 2}+z_{i}{ }_{i}}}
\end{aligned}
$$

As shown in Eq.(6)and(12),we can get Eq.(13)

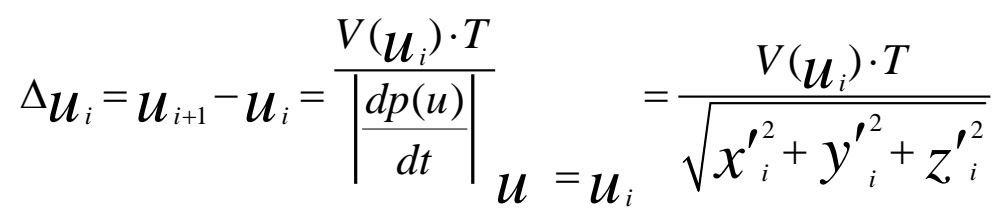

As shown in Eq.(13), the feed rate and interpolation period determines, in every interpolation cycle, chord length not edge, then interpolation cycle is the same.

\section{Cubic B-Spline curve chor error.}

If B curve interpolation can finished, the feed rate should keep costant as $V(t)$ during interpolation process. In Fig.1, $\rho_{i}$ is radius of curvature at $u_{i}, P\left(u_{i}\right)$ and $P\left(u_{i+1}\right)$ are the B curve interpolated .And $\delta_{i}$ is derived as

$$
\delta_{i}=\rho_{i}-\sqrt{\rho_{i}^{2}-\left(\frac{V\left(u_{i}\right) T}{2}\right)^{2}}
$$

The feed speed $V(t)$ corresponding to the chord ${ }^{\delta_{i}}$ is derived as 
If $\delta_{i}<\delta_{\text {max }}$, where $V_{\text {max }}\left(u_{i}\right)=\frac{2}{T} \sqrt{\rho^{2}-\left(\rho_{i}-\delta_{\max }\right)^{2}}=\frac{2}{T} \sqrt{\delta_{\max }\left(2 \rho_{i}-\delta_{\max }\right)}$

Eq.(13) expresses that B curve speed rate $V\left(u_{i}\right)$ should be included adaptively accrding to $\rho_{i}$ and $\delta_{i}$. So, ${ }^{\delta_{i}}$ can be represented the tolerance of the chord error. where $F$ is the feed speed command. Since B curve feed speed $V\left(u_{i}\right)$ is changed according to the radius of curvature, the following feed speed rule is proposed for parametric curves:

$$
V\left(u_{i}\right)= \begin{cases}F & \text { if } \delta_{i} \leq \delta_{\max } \\ \frac{2}{T} \sqrt{\rho^{2}-\left(\rho_{i}-\delta_{\max }\right)^{2}} & \text { otherwise }\end{cases}
$$

We should get B curve chord $\delta_{i}$, as shown in Tab.1

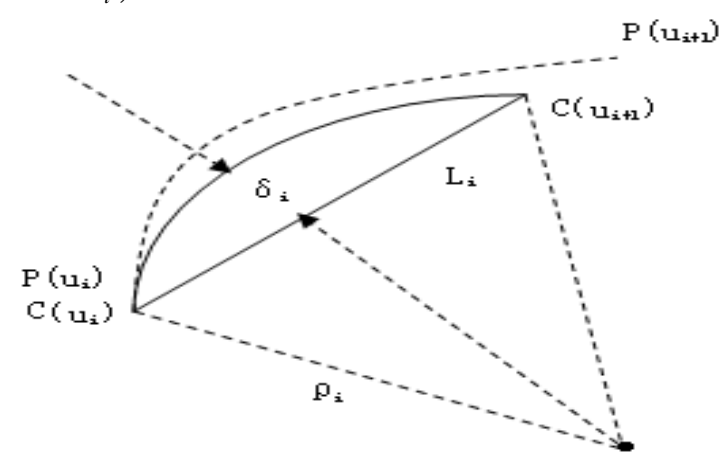

Fig.1 B curve chord $\delta_{i}$

\section{Example of Interpolation algorithm}

In the paper, the interpolation of a Cubic B-spline curve is utilized as an example to the modification algorithm, as shown in Tab 1.

The interpolation of Cubic B-spline curve parameters are as follows: the Cubic B-spline curve time in interpolation $T=1 \mathrm{~ms}$; the confined interpolation error is set as $\delta_{\text {max }}=1 \mu \mathrm{m}$; the command interpolation federate is given as $F=300 \mathrm{~mm} / \mathrm{s}$; the allowable acceleration and jerk $J=48000 \mathrm{~mm} / \mathrm{s}^{3}$.

\begin{tabular}{cccc} 
Tab. 1 The interpolation of Cubic B-spline curve parameters Table \\
\hline Parameter point & $\begin{array}{c}\text { control points of the } \\
\text { cubic B-Spline } \\
\text { curve(unit: mm) }\end{array}$ & $\begin{array}{c}\text { weight } \\
\text { vector }\end{array}$ & knot vector \\
\hline 1 & $(0,0,0)$ & 1 & \\
2 & $(1,1,2)$ & 1.2 & $(0,0$, \\
3 & $(2,1,2)$ & 2 & $0,0.451$ \\
4 & $(3,1.5,2)$ & 2 & 0.5485 \\
5 & $(3,2,2.5)$ & 1 & 0.6306, \\
6 & $(3.5,3,3)$ & 1 & 1,1, \\
7 & $(4,4.5,2.8)$ & 1.25 & $1,1)$ \\
8 & $(4,5,5$ & 0.85 & \\
9 & $(5,6,5.9)$ & 0.9 &
\end{tabular}

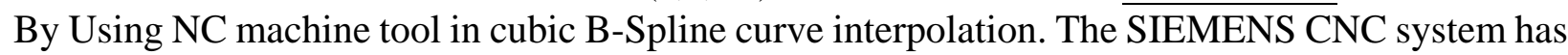
a wide and large share in the CNC system market ,and they make NC parameters as NC program command NC machine tool, Modification Cubic B-spline curve interpolation G code G06.2:

Cubic B-spline curve.txt, RT ' in the CNC machine tools complete interpolation. 


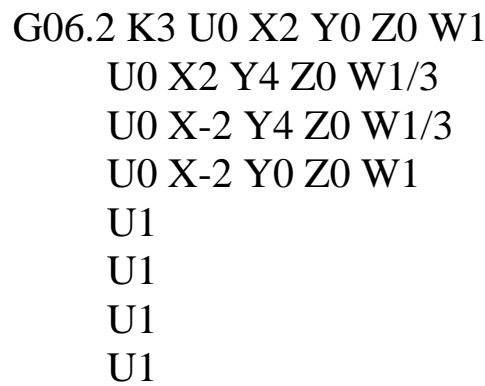

At now, G06.2 is the typical curve interpolation way for G code, such as SIEMENS CNC system. Figure 2 for NC machine tool in cubic B-Spline curve interpolation.

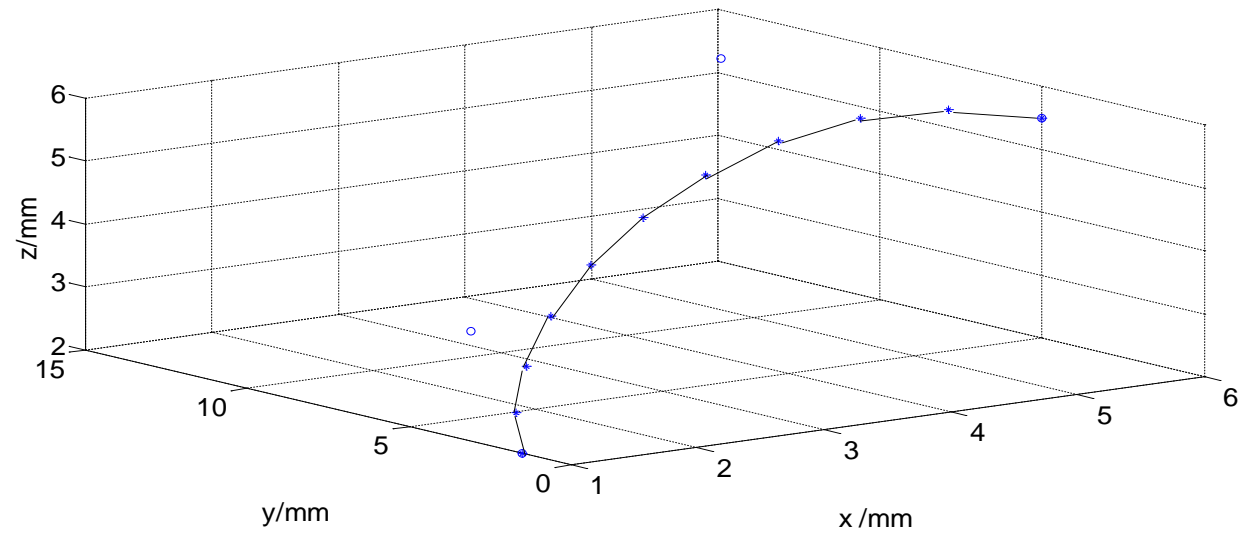

Fig. 2 CNC machine tools in complete interpolation example diagram

By Using computer soft in cubic B-Spline curve interpolation, as shown in Tab.2 and Figure 3.

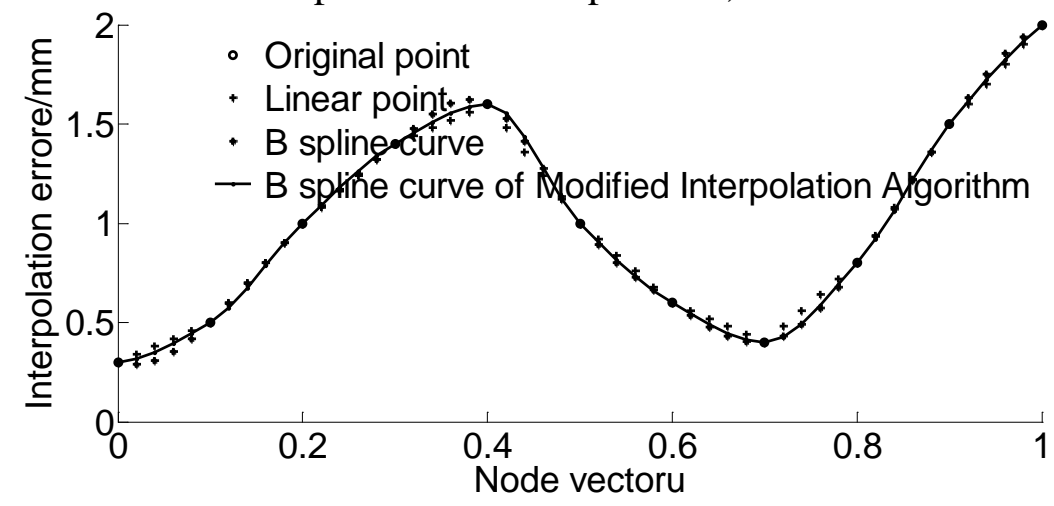

Fig. 3 cubic B-Spline curve with any interpolation point and original interpolation curve

Tab.2 Table analysis of interpolation of cubic B-Spline curve results

Step change $\triangle \mathrm{L}(\mathrm{mm}) \quad$ Interpolation step error e/mm Interpolation Chord error $\delta \quad \%$ )

\begin{tabular}{lccc}
\hline $\max$ & 0.452111 & 0.05521 & 0.0801 \\
$\min$ & 0.184511 & 0.0125 & 0 \\
\hline
\end{tabular}

As can be seen from Fig. 3 and table 2 shows, in the process of the interpolation, step changes, interpolation step error and interpolation chord error allows the error of maximum and minimum values, the interpolation chord error allowed values in the interpolation process significantly reduced, interpolation chord error max value $\delta(\%)$ is 0.0801 , which meet the expected to interpolation, i.e. to reduce the compensation error and interpolation step chord error. To verify the high efficiency and reliability of this cubic B-Spline curve interpolation algorithms are applied in the experiments to make a comparison .It can be seen that cubic B-Spline curve interpolation algorithm is feasible and efficent. 


\section{Summary}

In the paper, A novel on modification algorithm of Cubic B-spline curve interpolation is introduced. From the introduction and Mathematical analysis of the above mentioned the cubic B-Spline curve interpolation algorithm, we can see cubic B-Spline recursive formula of Taylor formula to expand the first order, two order expansions in the interpolation cycle of certain circumstances, interpolation and interpolation speed increment only about changing the interpolation increments can achieve interpolation correction purposes. The simulation results show that the proposed Cubic B-spline curve interpolator not only may also be in the CNC machine tools complete interpolation, but also meet the high-speed and high-accuracy NC machine tool require-ments of CNC systems. In addition ,NC machining time can be reduced. Also, being able to control Cubic $\mathrm{B}$-spline curve contour errors, improving the surface quality of workpieces. Implementation on NC machine has proven the feasibility of a developed interpolation algorithm.

\section{Acknowledgements}

This research was financially supported by the Science and Technology Major Project of “High-grade NC Machine Tools and Basic Manufacturing Equipment” (2013ZX04005-012).

\section{References}

[1] Fang Yi-Xiang, Liu Wen-Xue. Based on the geometric properties of the cubic uniform B-Spline curve structure description[J]. Journal of engineering graphics, 2 , pp.96-102, 2006.

[2] Zhang Wan-Jun, Hu Chi-Bing, Zhang Feng , et al . Honing machine motion control card three B spline curve method of interpolation arithmetic for CNC system [J]. Chinese Journal of Manufacturing Technology \& Machine Tool , 8(8), pp.40-43,August 2012.

[3] Zhang Wan-Jun, HU Chi-bing, WU Zai-xin, et,al. Research on modification algorithm of Three B Spline curve interpolation technology [J]. Chinese Journal of Manufacturing Technology \& Machine Tool , 2 pp.141-143,Feburary 2013.

[4] Zhang Wanjun, Zhang Feng , Zhang Guohua. Research on a algorithm of adaptive interpolation for NURBS curve. [J].Applied Mechanics and Materials , Vol. 687-691, pp.1600-1603, December 2014.

[5] Zhang Wan-Jun, Zhang Feng, Zhang Guohua. Research on modification algorithm of Cubic B-spline curve interpolation technology. [J].Applied Mechanics and Materials, Vol. 687-691, pp.1596-1599, December 2014.

[6] Kong Fan-Guo, Hao Shang-Hua, ZhongYan-Zhi. NURBS curve interpolation algorithm to achieve the VC [J]. China new technology and new products, 17, pp.8-9, 2009.

[7] Ye Bo-Sheng,Yang Shu-Zi. CNC system in cubic B-Spline curve interpolation method [J ]. China Mechanical Engineering, 9 ( 3) , 1998,pp. 42 - 43.

[8] Li He-Cheng, Wang Yu-Ping, An interpolation based genetic algorithm for sloving nonlinear bilevel programming problems.Chinese Journal of Computers, 31(6), pp.910-918, June 2008.

[9] Shpitalni M, Koren Y, Lo CC. Realtime curve interpolators. Computer- Aided Design, 26, 1994,pp.832-838.

[10] Huang JT, Yang DCH. A generalized interpolator for command generation of parametric curves in computer controlled machines. Japan/USA Symposium on Flexible Automation,1(1), pp.393-399, January1992.

[11] Zhang Wan-Jun, Zhang Feng, Zhang Wan-Liang. Research on a NURBS curve of timing / interrupt interpolation algorithm for CNC system [J]. Chinese Journal of Manufacturing Technology \& Machine Tool , 4(4), pp.40-43,April 2015. 